\title{
POST'S PROGRAMME FOR THE ERSHOV HIERARCHY
}

\author{
BAHAREH AFSHARI, GEORGE BARMPALIAS, \\ S. BARRY COOPER AND FRANK STEPHAN
}

\begin{abstract}
This paper extends Post's programme to finite levels of the Ershov hierarchy of $\Delta_{2}$ sets. Our initial characterisation, in the spirit of Post [24], of the degrees of the immune and hyperimmune $n$-enumerable sets leads to a number of results setting other immunity properties in the context of the Turing and wtt-degrees derived from the Ershov hierarchy. For instance, we show that any $n$-enumerable hyperhyperimmune set must be co-enumerable, for each $n \geq 2$. The situation with regard to the wtt-degrees is particularly interesting, as demonstrated by a range of results concerning the wtt-predecessors of hypersimple sets.

Finally, we give a number of results directed at characterising basic classes of $n$-enumerable degrees in terms of natural information content. For example, a 2-enumerable degree contains a 2-enumerable dense immune set iff it contains a 2-enumerable r-cohesive set iff it bounds a high enumerable set. This result is extended to a characterisation of $n$-enumerable degrees which bound high enumerable degrees. Furthermore, a characterisation for $n$-enumerable degrees bounding only low 2 enumerable degrees is given.
\end{abstract}

\section{INTRODUCTION}

Post's 1944 paper [24] initiated the investigation of the Turing degrees of enumerable sets ${ }^{1}$, and more generally, that of the relationship between natural information content and its Turing definability. His immediate goal was to show the existence of Turing degrees of enumerable sets intermediate between those of the empty set and the halting problem. In order to obtain his goal, he first defined simple, hypersimple and hyperhypersimple sets and later showed that they are incomplete for certain strong reducibilities. This approach did not carry over to Turing degrees. For example, hyperhypersimplicity always entails high Turing degree - hence leading to the upper and not the lower end of the hierarchy of enumerable Turing degrees.

Date: October 2006.

Key words and phrases. Turing degrees, Jump Classes, Ershov Hierarchy, Enumerable Sets, Immunity Properties, Cohesiveness, Post's Programme.

The first author was partially supported by an ORS (UK) award, and by the School of Mathematics, University of Leeds. The second two authors were supported by EPSRC grant No. GR /S28730/01, and by the NSFC Grand International Joint Project, No. 60310213, New Directions in the Theory and Applications of Models of Computation. The last author was supported in part by NUS grant number R252-000-212-112.

${ }^{1}$ That is, of computably enumerable (c.e.) or recursively enumerable (r.e.) sets. 
Nevertheless, many connections have been found between wtt-degrees and Turing degrees on one hand and structural properties like being simple on the other hand. In the present article, we investigate to what extent similar properties can be established for sets in Ershov's difference hierarchy.

The terminology and notation adopted here is a compromise between the individual authors of this paper, who variously follow the preferences of the textbooks written by Cooper [9], Cutland [10], Odifreddi [22] and Soare [28]. As a result, it is a bit similar to that of Downey and Lempp [13].

\section{The Degrees of IMmune AND hyPeRIMmune $n$-EnUmerable SetS}

We first generalise the main results of [1] and give more concise proofs for these results.

Theorem 1. If $A$ is n-enumerable, Turing incomputable and $n$ even then there are an n-enumerable immune set $B \equiv_{w t t} A$ and an n-enumerable hyperimmune set $C \equiv_{T} A$.

Proof. Since $n>1$ and $A$ is Turing incomputable $n$-enumerable, there is a Turing incomputable and enumerable set $E$ which is many-one reducible and thus wtt-reducible to $A$. Let

$\#_{E}(x)$ be the number of elements of $E$ up to $x$;

$c_{E}(x)$ be the time needed to enumerate all elements of $E$ up to $x$.

Using these, define

$$
\begin{aligned}
& B=\left\{\left(x, \#_{E}(x)\right): x \in A\right\} \text { and } \\
& C=\left\{\left(x, c_{E}(x)\right): x \in A\right\} .
\end{aligned}
$$

Clearly $B \leq_{w t t} A$ and $C \leq_{T} A$. Furthermore,

- $A \leq_{t t} B$ since $x \in A \Leftrightarrow \exists y \leq x+1[(x, y) \in B]$ and

- $A \leq_{T} C$ since $x \in A \Leftrightarrow \exists y \leq z[(x, y) \in C]$, where $z$ is the second half of the first pair $(u, z)$ found with $u>x \wedge(u, z) \in C$.

Note that the search for $(u, z)$ is successful since $A$ is Turing incomputable and infinite.

Let $F$ be an infinite subset of $B$. Then one can compute $E$ from $F$ : One can search for any given $x$ for the first pair $\left(y, \#_{E}(y)\right) \in F$ and afterwards enumerate the $\#_{E}(y)$ elements in $E$ up to $y$. If $x$ appears amongst these elements then $x \in E$; else $x \notin E$. Therefore $E$ is Turing reducible to $F$ and $F$ is not Turing computable. Thus $B$ is immune. 
Now assume that $D_{g(0)}, D_{g(1)}, \ldots$ is a sequence of disjoint finite sets each intersecting C. One can now construct the following function $h$ which Turingreduces to $g$ :

$$
\begin{aligned}
h(0)= & \text { the maximal } w \text { such that }(v, w) \in D_{g(0)} \text { for some } v ; \\
h(n+1)= & \text { the maximal } w \text { such that }(v, w) \in D_{g(m)} \text { for some } v, \\
& \text { where } m \text { is the first number such that } D_{g(m)} \text { does not } \\
& \text { intersect the rectangle }\{(x, y): x \leq n \wedge y \leq h(n)\} .
\end{aligned}
$$

It is easy to see that $h$ majorizes $c_{E}$. Thus one can compute $E$ relative to $h$ and relative to $g$. So $g$ is not a Turing computable function and $C$ is hyperimmune.

Theorem 2. If $A$ is n-enumerable with $n$ odd, then there is an n-enumerable co-immune set $B \equiv_{w t t} A$ and an n-enumerable co-hyperimmune set $C \equiv_{T} A$.

Proof. For the first part, note that there is a simple but not hypersimple set $E$ which is wtt-reducible to $A$. There is a Turing computable function $g$ defining a strong array $D_{g(0)}, D_{g(1)}, \ldots$ of disjoint sets such that $D_{g(x)}$ intersects the complement $\bar{E}$ of $E$ for every $x$ and every $D_{g(x)} \subseteq\{x, x+$ $1, x+2, \ldots\}$. Now let $B$ be the union of $E$ and all $D_{g(x)}$ with $x \in A$. Then $B$ is again $n$-enumerable and co-immune. Then $A \leq_{w t t} B$ since $x \in A \Leftrightarrow$ $D_{g(x)} \subseteq B$. Furthermore, $B \leq_{w t t} A$ since

$$
x \in B \Leftrightarrow x \in E \text {, or there is a } y \leq x \text { with } x \in D_{g(y)} \text { and } y \in A .
$$

This concludes the part of the proof referring to $B$.

For the construction of $C$, note that there is an $F$ Turing equivalent to $E$ such that $F$ is hypersimple and the complement $\left\{c_{0}, c_{1}, \ldots\right\}$ of $F$ is retraceable via a Turing computable function $g$, that is, $g\left(c_{x+1}\right)=c_{x}$ (by Dekker and Yates - see [22, Sections III.3 and III.4]). Now let $C=F \cup\left\{c_{x}\right.$ : $x \in A\}$. The set $C$ is $n$-enumerable and co-hyperimmune, as it is a superset of a hypersimple set.

\section{Hyperhyperimmunity AND the ERshov Hierarchy}

The purpose of this section is to show that hyperhyperimmunity in the finite levels of the difference hierarchy reduces to hyperhyperimmunity in the co-enumerable sets. This rules out any possibility of a straightforward extension of Martin's [20] characterisation of the high enumerable degrees in terms of appropriately complemented cohesive sets to higher levels of the Ershov hierarchy. We note that the situation for cohesiveness is more straightforward (easier proof) while for strong hyperhyperimmunity the difficulty is the same as for hyperhyperimmunity (which is what we deal with below). This is not surprising as by [6] any $\Delta_{2}^{0}$ set is hyperhyperimmune if and only if it is strongly hyperhyperimmune. We start with the following iterated version of Owings Splitting Theorem. 
Theorem 3. Suppose that $A, D$ are enumerable sets such that $\bar{A} \cup D$ is not enumerable. Then there are uniform sequences of enumerable sets $\left(E_{e}\right),\left(F_{e}\right)$ such that

(1) $\overline{E_{e}} \cup D, \overline{F_{e}} \cup D$ are not enumerable.

(2) for all $n, A=\left(\cup_{i<n} E_{i}\right) \cup F_{n}$

(3) $E_{i}$ are pairwise disjoint and for all $n, i<n, F_{n} \cap E_{i}=\emptyset$.

Proof. The Owings Splitting Theorem [23] says that given effective enumerations of $A, D$ we can uniformly define effective enumerations of $C_{0}, C_{1}$ such that $A=C_{0} \cup C_{1}, C_{0} \cap C_{1}=\emptyset$ and $\overline{C_{i}} \cup D$ are not enumerable. Our claim follows by iterating this procedure: since $\overline{C_{1}} \cup D$ is not enumerable we can apply the Owings procedure to get two disjoint enumerable sets $C_{10}, C_{11}$ such that $C_{1}=C_{10} \cup C_{11}$ and $\overline{C_{10}} \cup D, \overline{C_{11}} \cup D$ are not enumerable; we continue with $C_{11}$ and so on (see Figure 1).

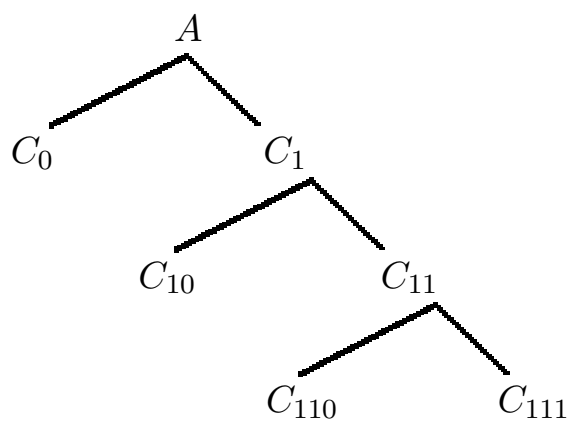

Figure 1: Iterating the Owings Splitting Theorem.

Define $F_{0}=A$ and for all $k \in \mathbb{N}$,

$$
\begin{aligned}
E_{k} & =C_{1^{k} 0} \\
F_{k} & =C_{1^{k}}
\end{aligned}
$$

It is clear that these enumerable sets have been obtained uniformly and so the sequences $\left(E_{k}\right),\left(F_{k}\right)$ are uniform sequences of enumerable sets. Moreover they have the properties (1) - (3) above since they have been obtained via Owings splittings as described previously.

Theorem 4. If $A$ is 2-enumerable and hyperhyperimmune then $A$ is coenumerable.

Proof. Fix a 2-enumerable approximation of $A$ and consider the set $P_{A}$ of the numbers that have appeared in $A$ at some stage of its approximation. Also, let $D_{A}$ be the set of numbers in $P_{A}$ which do not belong to $A$ (i.e. those which have entered and later been removed from $A$, see Figure 2 ). Note that both $P_{A}$ and $D_{A}$ are enumerable (the latter because once a number is extracted from $A$ it cannot enter again). 


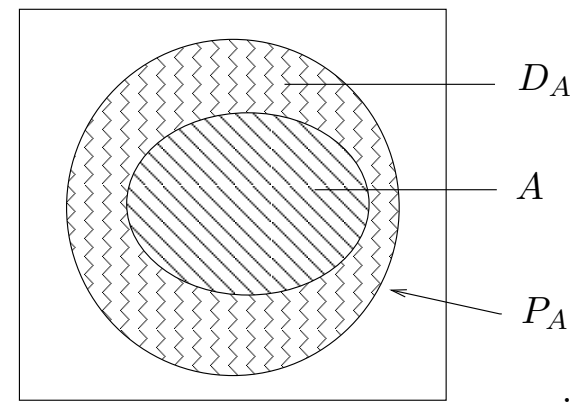

Figure 2: Approximation of a 2-enumerable set $A$

It is enough to show that if $A$ is not co-enumerable then there is a uniform sequence of finite pairwise disjoint enumerable sets such that each of its members intersects $A$. If $A$ is not co-enumerable, $\overline{P_{A}} \cup D_{A}$ cannot be enumerable. Now apply Theorem 3 and get a uniform sequence of pairwise disjoint sets $\left(E_{i}\right)$, subsets of $P_{A}$, such that $\overline{E_{i}} \cup D_{A}$ is not enumerable for any $i$. In particular, $E_{i} \nsubseteq D_{A}$ and so $E_{i} \cap A \neq \emptyset$ for all $i$. But $E_{i}$ are infinite, so define:

$$
\hat{E}_{i}[s]= \begin{cases}\hat{E}_{i}[s-1], & \text { if } \hat{E}_{i}[s-1] \cap A[s] \neq \emptyset ; \\ E_{i}[s], & \text { otherwise }\end{cases}
$$

where $[s]$ denotes the state of an object at the end of stage $s$ (the enumeration is based on that of $A$ and $\left.\left(E_{i}\right)\right)$. Since $E_{i} \cap A \neq \emptyset$, each $\hat{E}_{i}$ will be finite and $\hat{E}_{i} \cap A \neq \emptyset$ for all $i$.

This can now be extended to higher levels of the difference hierarchy.

Theorem 5. If $A$ is n-enumerable and hyperhyperimmune then $A$ is coenumerable.

Proof. Suppose $n>2$ and $A$ is $n$-enumerable and not $i$-enumerable for any $i<n$. By induction (and the previous theorem) we may assume that the claim holds for all $i<n$. It is enough to show that $A$ is not hyperhyperimmune. Suppose that it is for the sake of a contradiction. Consider an $n$-enumerable approximation to $A$ and the set $T_{A}$ of numbers that enter $A$ $\left\lceil\frac{n}{2}\right\rceil$ times $(\lceil x\rceil$ is the least integer $\geq x)$. Note that during the approximation any number can enter $A$ at most $\left\lceil\frac{n}{2}\right\rceil$ times.

Now for $n$ odd we immediately get a contradiction since (as a properly $n$-enumerable set) $A$ contains an infinite enumerable set and so cannot be hyperhyperimmune. If $n$ is even, $A \cap T_{A}$ is infinite (as $A$ is properly $n$ enumerable), 2-enumerable and hyperhyperimmune (as an infinite subset of a hyperhyperimmune set). By inductive hypothesis $A \cap T_{A}$ is co-enumerable and so $A$ is $(n-2)$-enumerable. Indeed, for an approximation with at most $n-2$ mind changes, run an enumeration of $\bar{A} \cup \overline{T_{A}}$ and of the $n$ enumerable approximation of $A$ with the following modification: when a 
number is already subject to $n-3$ mind changes (and so is currently valued 1) we only change it to 0 if

- our $n$-enumerable approximation requires it and

- the number has appeared in $\bar{A} \cup \overline{T_{A}}$

(and after that this number does not change anymore). This is an $(n-2)$ enumerable approximation. It is not hard to see that the set we obtain is $A$. This gives a contradiction since we assumed that $A$ is not $(n-2)$-enumerable.

Corollary 6. If $A$ is n-enumerable and cohesive then $A$ is co-enumerable.

\section{Hypersimple Weak truth table Degrees}

Although Post [24] completely characterised the Turing degrees of simple and hypersimple sets, Theorems 1 and 2 above still leave some basic gaps in our knowledge of the wtt-degrees of such sets.

A few facts about hypersimple sets in the wtt degrees can be found at $[2,3,4,12]$. In particular, every hypersimple wtt degree is noncuppable in the enumerable wtt degrees [12]. Also, the enumerable hypersimple-free wtt degrees are dense in the enumerable wtt degrees [4]. It follows that hypersimple wtt degrees are not the only noncuppable enumerable wtt degrees. In [3] (a published version of the last chapter of [2]) other facts (e.g. existence of many hypersimple free upper cones in the enumerable wtt degrees) are shown and some connections with cuts of computable linear orderings of $\mathbb{N}$ (of type $\omega+\omega^{*}$ ) are established. Recall that a $\Delta_{2}^{0}$ set $A$ is called superlow if $A^{\prime} \leq_{\mathrm{wtt}} \emptyset^{\prime}$ (which is equivalent to $A^{\prime} \leq_{\mathrm{tt}} \emptyset^{\prime}$ ). Bickford and Mills [5] (also see [21]) show that there are two superlow enumerable sets which join to a set Turing above the complete set but no superlow enumerable set is cuppable in the wtt enumerable degrees. Theorem 7 is stronger than the latter statement because of Theorem 16. In other words there is a noncuppable enumerable wtt degree which is not bounded by any hypersimple wtt degree.

Theorem 7. Every superlow enumerable wtt degree is bounded by a hypersimple wtt degree while there are low enumerable wtt degrees which are not bounded by any hypersimple wtt degree.

Proof. An inspection of the direct construction (finite injury) in [3] of a enumerable wtt degree which does not bound any hypersimple wtt degrees it is clear that such degrees can be built low. For the other part assume that $A$ is enumerable such that $A^{\prime}$ is $\omega$-enumerable and build a hypersimple $B$ such that $A \leq_{\mathrm{wtt}} B$. The hypersimplicity requirements are

$$
H_{e}:\left(D_{i}^{e}\right) \text { total } \Rightarrow \exists n\left(D_{n}^{e} \cap \bar{B}=\emptyset\right)
$$

where $D^{e}=\left(D_{i}^{e}\right)$ is the eth strong disjoint array in some effective enumeration. For the coding we consider a monotonic effective enumeration $\phi(0), \phi(1), \ldots$ of $\mathbb{N}^{[0]}$. The construction will ensure that if $n \searrow A$ at some stage, there will be a later stage where $B \uparrow \phi(n)$ changes; thus $A \leq_{\mathrm{wtt}} B$. 
There is a conflict which comes from the hypersimplicity requirements which try to take up too many codes, thus threatening the reduction (see [3]). We deal with this by using the superlowness of $A$ to make the coding more flexible. We build a weak array $\left(U_{e}\right)$ during the construction and by the recursion theorem we may assume that we know an index of it. We enumerate finite strings into $U_{e}$ (which corresponds to $H_{e}$ ) intended as guesses about initial segments of $A$, in such a way that we do not put a segment unless all existing ones have been proved wrong. Then we can ask the question 'does $U_{e}$ contain an initial segment of $A$ ?' which is $\Sigma_{1}^{A}$ and is many-one reducible to $A^{\prime}$. Since $A^{\prime}$ is $\omega$-enumerable we may assume (also because of the recursion theorem) that we have computable functions $g(e, s)$ and $f(e)$ such that

$$
\lim _{s} g(e, s)=1 \Longleftrightarrow U_{e} \text { does not contain an initial segment of } A
$$

and the mind-changes for $g$ on argument $e$ are less than $f(e)$. Say that $g$ is a $\{0,1\}$-valued function.

The construction with one $H_{e}$ requirement consists of the standard coding (if $n \searrow A$ put $\phi(n) \searrow B$, unless $\phi(n)$ is already in $B$ in which case see below) and the action for $H_{e}$ which requires attention if

- for all $i, D_{i}^{e} \downarrow \Rightarrow D_{i}^{e} \cap \bar{B}$

- for some $i$ the term $D_{i}^{e}$ is defined and $\left|\left[0, \min D_{i}^{e}\right) \cap \mathbb{N}^{[e]} \cap \bar{B}\right|>f(e)$.

Without loss of generality we can assume that each $D_{i}^{e}$ is a segment of the form $(\phi(p), \phi(q)]$ If this happens (say at stage $s), H_{e}$ acts by putting (the elements of $) D_{i}^{e}=(\phi(p), \phi(q)]$ into $B$ and declaring that the numbers in $(p, s]$ are $e$-coloured. From then on the coding will continue as before with the only exception the numbers in $(p, s]$ (whose natural codes, their $\phi$-codes, may have been consumed). If such a number later enters $A$ we run the recovery process for $n$ which either observes a later $A$-enumeration $m \searrow A$ of a non-coloured $m \leq n$ (in which case we just have to put $\phi(m) \searrow B$ and the coding is done) or it extracts a new 'yes' answer from the function $g$ about our question of whether $U_{e}$ contains initial segments of $A$. In the latter case we put into $B$ a number of $\mathbb{N}^{[e]}$ which is less than $\phi(n)$. The fact that $g$ can change its mind about $U_{e}$ less than $f(e)$ times shows that we will never be short of codes and thus the coding works.

In the full construction an $H$-strategy may, during its main action, enumerate into $B$ some numbers of $\mathbb{N}^{[e]}$ where $H_{e}$ is a lower priority strategy which has already acted (and thus it may need these numbers for a recovery process). However this is only an apparent conflict since from that point on there will be no $e$-coloured numbers and no later recovery process will have to deal with $e$-coloured numbers. Another issue is that we need to ensure that $\mathbb{N}^{[e]} \cap \bar{B}$ is infinite for all $e$. We do this by setting $\mathbb{N}^{[e]} \cap \bar{B}=\left\{b_{0}^{e}, b_{1}^{e}, \ldots\right\}$ (at all stages) and demanding that $H_{e}$ does not enumerate any number in the interval $\left[0, \max _{i \leq e} b_{e}^{i}\right]$. We assume a universal enumeration of enumerable sets etc. and based on these stages we define the construction. 
Construction. Start from stage 0 and do the following.

Step 1. If some (least) $n$ has just been enumerated into $A$ start the recovery process for $n$ : If $n$ is not coloured (i.e. $\phi(n) \notin B$ ) put $\phi(n) \searrow B$ and go to step 2. Otherwise, if $n$ is $e$-coloured, $\left(p, s_{0}\right)$ is the $e$-coloured interval and $s_{1}$ the current stage, find $s_{2}>s_{1}$ such that $g\left(e, s_{2}\right) \downarrow \neq 1$ or some $m<n$ is enumerated into $A$. In the latter case run the recovery process for $m$ and in the former case do the following: put $A \uparrow s_{0} \searrow U_{e}$ and find a stage $s_{3}>s_{2}$ such that either $g\left(e, s_{2}\right) \downarrow=1$ or $A \uparrow s_{0}$ changes.

(a) In the former case put into $B$ the least number of $\mathbb{N}^{[e]} \cap \bar{B}\lceil\phi(p)$ which is larger than $b_{e}^{i}$ for all $i \leq e$ (if such exists) and go to step 2.

(b) If some $m<n$ is enumerated into $A$, run the recovery process for $m$.

(c) Otherwise (i.e. if some number of $\left(n, s_{0}\right)$ is enumerated in $A$ ) re-run the recovery process for $n$.

Step 2. Let $e$ be the least (if any) such that $H_{e}$ requires attention i.e. the following conditions hold:

- for all $i, D_{i}^{e} \downarrow \Rightarrow D_{i}^{e} \cap \bar{B}$

- for some $i$ the term $D_{i}^{e}$ is defined and there are more than $f(e)$ numbers in $\bar{B} \cap \mathbb{N}^{[e]}$ which are less than $\min D_{i}^{e}$ and larger than $b_{e}^{i}$ for all $i \leq e$.

If $s$ is the current stage and $D_{i}^{e}=(\phi(p), \phi(q)]$, put $(\phi(p), \phi(s)] \searrow B$ and say that $(p, s]$ is $e$-coloured. Go to the next stage and to step 1.

We verify the construction.

Lemma 8. If g has the property (2) then the construction goes through steps 1,2 infinitely many times.

Proof. We only need to check that it does not get stuck at step 1 . Note that if $\left(p, s_{0}\right)$ is the $e$-coloured interval for some $e, U_{e}$ contains only approximations to $A \uparrow s_{0}$ (and so, segments of a fixed length). By construction whenever we ask for a later stage $s$ such that $g(e, s) \neq 1$, some $e$-number has entered $A$ and so all strings in $U_{e}$ have been shown to be incompatible with $A$. Hence these searches will halt by the properties of $g$. The searches which ask for a later stage $s$ with $g(e, s)=1$ or all strings in $U_{e}$ are incompatible with $A_{s}$ will also halt due to $(2)$. Since we only run the recovery process for non-increasing sequence of numbers in $[0, n]$ the recovery process will settle on a final number $m \leq n$ and since we put in $U_{e}$ segments of a fixed length (the maximum member of the $e$-coloured interval) $g(e)$ will settle on a positive answer. So we will pass to step 2 .

By the Recursion Theorem we may assume that $g$ satisfies (2) and, as before, the mind-changes for $g$ on argument $e$ are less than $f(e)$.

Lemma 9. For all $i, e, \lim _{s} b_{e}^{i}[s]<\infty$. 
Proof. By explicit rules in the construction $b_{e}^{i}$ can only be moved by the action of some $H_{k}$ with $k<\max (i, e)$. Each $H_{k}$ acts at most once via step 2 and at most finitely many $k$-coloured numbers (the members of the possible $k$-coloured interval) can be enumerated by a recovery process. Hence each $b_{e}^{i}$ can be moved finitely many times.

When a number is $e$-coloured and later becomes $e^{\prime}$-coloured (for $e^{\prime} \neq e$ ), it stops being e-coloured.

Lemma 10. If at some stage there are e-coloured numbers and $\left(\phi(p), s_{0}\right]$ is the interval enumerated into $B$ by $H_{e}$,

$$
\left|\mathbb{N}^{[e]} \cap \bar{B} \cap\left(\max _{i \leq e} b_{e}^{i}, \phi(p)\right]\right|>f(e)-c(e)
$$

where $c(e)$ is the (current) number of mind-changes of $g$ from 0 to 1.

Proof. Since there are $e$-coloured numbers, no strategy other than $H_{e}$ has enumerated numbers of $\mathbb{N}^{[e]} \uparrow \phi(p)$ into $B$ since the attack of $H_{e}$. In particular, the markers $b_{e}^{i}$ for $i \leq e$ have not been moved since that stage. When $H_{e}$ attacked via step 2 there were more than $f(e)$ numbers in $\mathbb{N}^{[e]} \cap \bar{B} \cap\left(\max _{i \leq e} b_{e}^{i}, \phi(p)\right]$. From that point on every time such a number was enumerated (by a recovery process for an $e$-coloured number) a new mind-change of $g$ from 0 to 1 is observed. So (3) follows by induction.

Lemma 11. $A \leq_{w t t} B$.

Proof. First we show that the coding works, i.e. that whenever step 1 of the construction asks for a suitable number of $\bar{B}$ to be enumerated in $B$, such a number exists. Indeed, if it asks to enumerate some $\phi(k)$ the number $k$ is not coloured and $k$ has just appeared in $A$ hence $\phi(k)$ is not yet in $B$. Otherwise there would be $e$-coloured numbers for some $e$ and it would ask to enumerate a number of $\mathbb{N}^{[e]} \cap \bar{B} \cap\left(\max _{i \leq e} b_{e}^{i}, \phi(p)\right]$. By Lemma 10 it is enough to show that $c(e) \leq f(e)$ at all stages. But this follows from the properties of $f$ and $g$.

It follows from the construction that whenever a number appears in $A$ step 2 is not accessed until an enumeration into $B$ of a number $\leq \phi(n)$ is requested. Since all such requests are fulfilled and by Lemma 8 the construction goes through steps 1,2 infinitely many times, every change of $A \uparrow n$ is followed by a change of $B\left\lceil\phi(n)\right.$ and so $A \leq_{\text {wtt }} B$.

Lemma 12. B is hypersimple.

Proof. It suffices to show that each $H_{e}$ is satisfied. So suppose that $\left(D_{i}^{e}\right)$ is total. By Lemma 9 the set $\mathbb{N}^{[e]} \cap \bar{B}$ is infinite and so $H_{e}$ will require attention infinitely often unless $D_{i}^{e} \cap \bar{B}=\emptyset$ for some $i$. As usual there will be a stage after which no higher priority requirement requires attention and so $H_{e}$ will be satisfied.

These lemmas conclude the proof. 
The next result gives some sufficient criterion (without any well-established name) for an enumerable set to be wtt-reducible to a hypersimple set. This criterion is quite general, as Ishmukhametov [15] showed that every enumerable set which has a strong minimal cover satisfies this criterion. It also generalises several of the known traceability-constraints investigated by various authors. Some $\operatorname{low}_{2}$ but not all low sets satisfy this criterion. Note however that every superlow enumerable set satisfies it and so Theorem 7 follows from the one below.

Theorem 13. Assume that $A$ is enumerable and satisfies that for every function $u \leq_{T}$ A there exist computable functions $f, g$ such that $u(x) \in$ $W_{g(x)}$ and $\left|W_{g(x)}\right| \leq f(x)$ for all $x$. Then there is a hypersimple set $B$ with $A \leq_{w t t} B \leq_{T} A$.

Proof. Let $A_{s}$ be the set of elements enumerated into $A$ within $s$ stages. Furthermore, for input $x$ let

$$
\begin{aligned}
c_{A}(x) & =\min \left\{s>x: \forall y \leq x\left(A_{s}(y)=A(y)\right)\right\} ; \\
u(x) & =c_{A}\left(c_{A}(x)\right) .
\end{aligned}
$$

By assumption, there are increasing computable functions $f, g$ such that $u(x) \in W_{g(x)}$ for all $x \in A$ and $\left|W_{g(x)}\right|<f(x)$ for all $x$. Note that one can approximate $u(x)$ from below for all $x$, thus one can assume that $u(x)=$ $\max \left(W_{g(x)}\right)$ for all $x$.

Let $I_{0}, I_{1}, I_{2}, \ldots$ be a partition of the natural numbers into intervals where the length of $I_{x}$ is $f(x)$.

One defines an enumerable set $B$ being the domain of $\psi$. Given $x$, let $y$ be the index of the interval with $x \in I_{y}$. Now $x \in B$ if one of the following two cases applies; $\psi(x)$ is chosen according to that of the two cases which is found first to apply; $\psi(x)$ is undefined if none of the cases applies:

(a) There are $m, s, z$ with $m \leq z<y \leq s, m \in A_{s+1}-A_{s}$ and $I_{z} \not B_{s}$ : then $I_{y} \subseteq B_{s+1}$ and $\psi(x)=s$;

(b) $\left|W_{g(y)}\right|>x-\min \left(I_{y}\right)$ : then $\psi(x)$ is the $\left(x-\min \left(I_{y}\right)+1\right)$-st element enumerated into $W_{g(y)}$.

Now the wtt-reduction from $A$ to $B$ is that $x \in A$ iff $x \in A_{s}$ for $s$ being the maximum of $\psi$ on $I_{0} \cup I_{1} \cup \ldots \cup I_{x}$. Note that this maximum can be computed by the wtt-reduction as the domain $B$ on these intervals is provided.

To see that this is correct, take any value $x$. Let $n$ be the largest stage such that some of the intervals $I_{0}, I_{1}, \ldots, I_{x}$ was enumerated into $B$ according case (a) and let $z$ be the parameter used in this stage - more precisely, the least possible value this parameter can take. Then $I_{z}$ is never enumerated according to condition (a) and $\psi$ is defined on $I_{z}$ occording to case (b). So $\psi(a)=c_{A}\left(c_{A}(z)\right)$ for some $a \in B \cap I_{z}$.

If $n \geq x$ then $c_{A}\left(c_{A}(z)\right) \geq c_{A}(n) \geq c_{A}(x)$ and $s \geq c_{A}\left(c_{A}(z)\right) \geq c_{A}(x)$. If $n<x$ then $I_{x}$ is never enumerated into $B$ according to case (a) and some $a \in B \cap I_{x}$ takes the value $c_{A}\left(c_{A}(x)\right)$. Hence again $s \geq c_{A}(x)$. In both cases, $x \in A$ iff $x \in A_{s}$. 
For proving the reverse reduction $B \leq_{T} A$, note that given any interval $I_{y}$ and $x \in I_{y}$, one can find using $A$ as an oracle the last stage $n$ in which some of the intervals $I_{0}, I_{1}, \ldots, I_{y}$ is enumerated into $B$ according to case (a). If $I_{y} \subseteq B_{n+1}$ then $B(x)=1$. Otherwise, note that the function $u$ is approximable from below as

$$
u(y)=\max \left\{c_{A_{0}}\left(c_{A_{0}}(y)\right), c_{A_{1}}\left(c_{1_{0}}(y)\right), c_{A_{2}}\left(c_{A_{2}}(y)\right), \ldots\right\} .
$$

Without loss of generality, $W_{g(y)}$ is defined such that $u(y)$ is the last member enumerated into $W_{g(y)}$. As $u \leq_{T} A$, one can get the whole list of $W_{g(y)}$ using the oracle $A$ and determine the last stage $s$ where $B$ is adjusted on $I_{y}$ in order to get a new element of $W_{g(y)}$ be into the range of $\psi$. Then $x \in B \Leftrightarrow x \in B_{s+1}$. Hence $B \leq_{T} A$.

Assume now by way of contradiction that $B$ is not hypersimple. Then there is a computable function $h$ such that for every $n$ there is an $m$ with $n \leq$ $m<h(n)$ and $I_{m} \not B B$. As a consequence, there are two such intervals with an index between $n$ and $h(h(n))$. If now $m \in A_{s+1}-A_{s}$ then $h(h(m))>s$ because there is at most one number $z$ with $m \leq z \leq s$ such that $I_{z} \nsubseteq B_{s+1}$ due to the way the enumeration of $B$ is defined in case (a) and between $m$ and $h(h(m))-1$ there are two indices of such intervals. Thus $m \in A \Leftrightarrow m \in$ $A_{h(h(m))}$ in contradiction to the fact that $A$ is Turing incomputable. So $B$ is hypersimple.

Corollary 14. If $A$ is as in Theorem 13 and $D \leq_{T} A$ then $D$ is wtt-reducible to a hypersimple set.

Proof. Given $A$ as in Theorem 13 and $D \leq_{T} A$, there is a Turing reduction and an $A$-Turing computable bound $d$ which can be approximated from below such that the computation of $D(x)$ needs less than $d(x)$ steps and does not query any $y \geq d(x)$. Without loss of generality, $x \leq d(x) \leq d(x+1)$ for all $x$.

The construction of $B$ in the proof of Theorem 13 is adapted in one point, namely

$$
u(x)=c_{A}\left(d\left(c_{A}(x)\right)\right)
$$

and all further changes are consequences of this change. That is, the functions $f, g$ and intervals $I_{0}, I_{1}, I_{2}, \ldots$ have to be adapted to this changed definition of $u$. Then there is an approximation $D_{s}$ to $D$ such that

$$
\forall x \forall s\left[\left(\forall y \leq x \forall a \in B \cap I_{y}(\psi(a) \leq s)\right) \Rightarrow\left(D(x)=D_{s}(x)\right)\right]
$$

and therefore $D \leq \leq_{w t t} B$. Furthermore, the proof that $B$ is hypersimple is analogous to the one in Theorem 13. The direction $B \leq_{T} D$ can in general not be saved as $D$ might have a Turing degree which is not enumerable.

Remark 15. An application of Theorem 13 is that every Turing incomputable $H$-trivial enumerable Turing degree contains for every enumerable set $A$ a further enumerable set $B \geq_{w t t} A$ such that $B$ is hypersimple. Here a set is $H$-trivial iff there is a constant $c$ such that for every $n$ the prefix-free 
Kolmogorov complexities $H(A(0) A(1) \ldots A(n))$ and $H(n)$ do not differ by more than $c$.

The property given in Theorem 13 is near to being strongly contiguous but not the same. Note that the property of Theorem 13 is inherited downwards while being strongly contiguous is not inherited downwards [26], see also [22, Exercise X.8.15].

But for those enumerable sets whose Turing degree is bounded by a strongly contiguous enumerable degree, it is easy to see that they are wttreducible to a hypersimple set as well: So, given an enumerable set $A$ which is bounded by a strongly continguous degree, there is a hypersimple set $B$ in this strongly contiguous degree. Then $A \leq_{w t t} B$ as observed by Odifreddi [22, Exercise X.8.11].

Say that a wtt degree is noncuppable in the structure of enumerable wtt degrees if its join with any wtt incomplete enumerable degree is wtt incomplete. In other words, an enumerable set $A$ is noncuppable iff $K \mathbb{L}_{w t t} A$ and for all enumerable sets $B, K \leq_{w t t} A \oplus B$ implies $K \leq_{w t t} B$. Both types of wtt degrees exist. For example Sacks Splitting Theorem splits the halting problem $K$ into two disjoint wtt-incomplete enumerable sets $A$ and $B$ whose join is wtt-equivalent to $K$; hence they are wtt cuppable. On the other hand, it is well-known that noncuppable wtt degrees also exist and indeed, the next result provides such a degree with some interesting additional properties.

Theorem 16. In the enumerable wtt degrees there is a low noncuppable degree which is not bounded by any hypersimple degree.

Proof. For the construction one splits the natural numbers into intervals $I_{0}, I_{1}, I_{2}, \ldots$ such that the length of each interval $I_{n}$ is an interval of the form $\left\{a, a+1, \ldots, a+(n+3)^{2}\right\}$ for some $a$. Now a priority construction is done to satisfy the following requirements:

- Requirement $R_{2 e}$ : For the $e$-th triple $(f, g, W)$ of a candidate wttreduction with a computable bound function $f$ and an evaluation function $g$ to an enumerable set $W$ then the requirement tries to get hold of an interval $I_{x}$ such that at every stage $s$ it holds that $\min \left(I_{x}\right)>r_{k, s}$ for all $k<2 e, x>e, f$ is defined and strictly monotonic increasing on $0,1,2, \ldots, \max \left(I_{x}\right)$, the values $x, x+1, x+2$, $\ldots, f\left(\max \left(I_{x}\right)\right)$ are all enumerated into $W$. If no candidate $I_{x}$ is found at stage $s$, then $r_{2 e, s}=\max \left\{u: u=1 \vee u=1+r_{k, s}\right.$ for some $k<s\}$. If such a candidate $I_{x}$ is found at stage $s$, then $r_{2 e, s}=$ $\max \left(I_{x}\right)$ and the next goal is to find an $y \leq \max \left(I_{x}\right)$ with either $A(y) \neq g(W(0), W(1), \ldots, W(f(y)))$ or $g(W(0), W(1), \ldots, W(f(y)))$ being undefined. This can be satisfied by enumerating up to $x+1$ elements of $I_{x}$ into $A$ in order to satisfy this; note that $W$ changes in the queried region at most $x$ times as $x, x+1, x+2, \ldots, f\left(\max \left(I_{x}\right)\right)$ are already in $W$ when the requirement picks $I_{x}$. 
- Requirement $R_{2 e+1}$ only updates restraints in order to spread the intervals out but does not enumerate any elements. It satisfies that $r_{2 e+1, s}$ is the maximal use of any computation $\varphi_{i}^{A_{s}}(j)$ terminating within time $s$ where $i, j \leq r_{2 e, s}$.

As all requirements ask only for finitely many activity, they are satisfied eventually. Note that the search for the interval $I_{x}$ at requirement $R_{2 e}$ might not terminate if the corresponding set $W$ is not hypersimple; in this case it just happens that $r_{2 e, s}$ will converge to some value larger than the limit of all $r_{k, s}$ with $k<2 e$ but nothing else will happen.

Otherwise the corresponding interval $I_{x}$ will eventually be found due to $W$ being hypersimple and higher priority requirements moving their restraints out only finitely often. As only requirements $R_{2 e^{\prime}}$ with $e^{\prime}<x$ access the interval and as it has $(x+2)^{2}$ elements, it happens that each of these requirements enumerate up to $x+1$ elements of this interval into $A$ and thus there will still be at least $x$ not yet enumerated elements when requirement $R_{2 e}$ puts its restraint onto $I_{x}$. From that time on, there can at most $x$ elements below $f\left(\max \left(I_{x}\right)\right)$ be enumerated into $W$.

So for each stage $s$ where either $I_{x}$ was selected or an element below $x$ was enumerated into $W$, one can find a $y \in I_{x}-A_{s}$ and then monitor how $g\left(W_{s}(0) W_{s}(1) \ldots W_{s}(f(y))\right)$ is behaving on the $s$-th approximation $W_{s}$ of $W$. If it converges to 0 , one makes the computed value false by enumerating $y$ into $A$; otherwise one keeps $y$ out of $A$.

It is easy to see that the requirements $R_{2 e+1}$ enforce that $A$ is low; indeed, such types of requirements are the standard way to make a set low.

To show that the noncuppability requirements are satisfied one can use Kolmogorov complexity (for background we refer to [11]). Assume that $A \oplus B$ is wtt-complete. Then random sets like Chaitin's $\Omega$ are wtt-reducible to $A \oplus B$ and thus there is a strictly increasing computable function $h$ such that $C(A(0) B(0) A(1) B(1) \ldots A(y) B(y))>x$ for all $x$ and all $y \geq h(x)$ where $C$ is the plain Kolmogorov complexity. Now it is shown that there is an other function $\tilde{h}$ such that, for all $x$,

$$
C(B(0) B(1) \ldots B(\tilde{h}(\tilde{h}(x))))>x
$$

as well. Thus $B$ is an enumerable and dnr set, hence wtt-complete. So $A$ joins to $K$ only with wtt-complete enumerable sets $B$ and so $A$ is not cuppable (within the enumerable wtt-degrees).

Let $r_{k, \infty}$ be the supremum of $r_{k, s}$ over all $s$. Let $\tilde{h}(x)=h\left((2 x+c)^{6}\right)$ where $c$, using the Fixed-Point Theorem, is chosen such that $c$ is sufficiently large, $c$ satisfies $c>r_{0, \infty}$ and $c$ is the index of a function $\varphi_{c}^{E}$ which has on input $x$ computes $E(\tilde{h}(\tilde{h}(x)))$ and thus has the use $\tilde{h}(\tilde{h}(x))$, independently of what the set $E$ is. Now let $e$ be the minimal number such that $r_{2 e+1, \infty} \geq \tilde{h}(x)$. By the choice of $c$ and the form of Requirement $R_{2 e+1}$, one has $r_{2 e+1, \infty} \geq$ $\tilde{h}(\tilde{h}(x))$ whenever $r_{2 e, \infty} \geq x$. Thus one can choose $y \in\{x, \tilde{h}(x)\}$ such that $r_{2 e, \infty} \leq y \leq \tilde{h}(y) \leq r_{2 e+1, \infty}$. There are only $r_{2 e, \infty}^{3}$ many stages $s$ 
in which $r_{2 e+1, s+1}>r_{2 e+1, s}$. After the last of these stages $s, A$ does not make any further change between $r_{2 e, \infty}$ and $r_{2 e+1, \infty}$. So one can describe $A(0) A(1) \ldots A(\tilde{h}(y))$ by $y, e$ and a number $a \in\left\{0,1,2, \ldots, y^{3}\right\}$ such that $s$ is the $a$-th member of $\left\{t: r_{2 e+1, t+1}>r_{2 e+1, t}\right\}$ and the number $b$ of elements in $A$ between 0 and $y$. $b$ is also bounded by $y$. Hence

$$
\begin{aligned}
C(A(0) A(1) \ldots A(\tilde{h}(y))) & \leq(y+c)^{5} ; \\
C(A(0) B(0) A(1) B(1) \ldots A(\tilde{h}(y)) B(\tilde{h}(y))) & \geq(y+c)^{6} ; \\
C(B(0) B(1) \ldots B(\tilde{h}(y))) & \geq 2 y+c ;
\end{aligned}
$$

provided that the constant $c$ had been chosen large enough above (what was possible). As $y \in\{x, \tilde{h}(x)\}$, it follows that

$$
C(B(0) B(1) \ldots B(\tilde{h}(\tilde{h}(x)))) \geq x
$$

which again needs that $c$ is sufficiently large. Altogether, one has that $B$ is wtt-complete.

A more conventional proof of Theorem 16, a priority tree argument, is possible via a combination of the hypersimple avoidance strategies of $[2,3]$ and a wtt adaptation of the noncupping tree strategies of [29].

\section{ERshov Hierarchy AND Jump-Classes}

One starting point for the present paper was the aim of characterising, via natural criteria, the jump classes of sets in the Ershov hierarchy. Significantly, when attempting to extract results corresponding to those for the first level of the hierarchy, it turns out that much more important than the jump class of the actual set $A$ is the question of which jump classes the enumerable sets $E \leq_{T} A$ occupy. The following two results lead to characterisations of the sets $A$ in the Ershov hierarchy with respect to the question of whether they bound a high or a non- low $_{2}$ enumerable degree. Combining these with recent results concerning isolated 2-enumerable degrees (see below), they set some surprising limits on the extent to which the work of Martin and others for the high enumerable degrees can be extended.

Definition 17 (Martin [19]). A set $A=\left\{a_{0}, a_{1}, a_{2}, \ldots\right\}$ is dense immune iff there is no computable function $f$ with $f(n) \geq a_{n}$ for infinitely many $n$; a dense simple set is an enumerable set with a dense immune complement [22, Exercise III.3.9].

Definition 18. A set $A$ is $r$-cohesive iff for every decidable set $B$, either $A-B$ or $A \cap B$ is finite. A set is $r$-maximal iff it is enumerable and has an $r$-cohesive complement.

Recall that an introreducible set is a set which is Turing reducible to every infinite subset of itself. As the next result will essentially need that there are introreducible sets with an r-maximal but not maximal complement below any given high enumerable Turing degree, the existence of such sets has to been proven first. 
Proposition 19. Let $D$ be an enumerable set of high Turing degree. Then there is an introreducible set $E \leq_{T} D$ such that $E$ is r-cohesive, $E$ is not cohesive and $\bar{E}$ is enumerable.

Proof. Without loss of generality, it can be assumed that the function mapping $x$ to the first stage $s$ such that $D_{s}(y)=D(y)$ for all $y<x$ dominates every computable function. Such a set $D$ is called enumeration-dominant and it exists in every high enumerable Turing degree [25]. This property is needed to make sure that the permitting below works as desired and that the permission to update the $e$-state beyond a certain point is given for almost all markers who require this.

First one splits the natural numbers inductively into intervals $I_{0}, I_{1}, I_{2}, \ldots$ such that $I_{0}=\{0,1,2,3,4,5,6,7,8\}$ and $I_{n+1}=\left\{\max \left(I_{n}\right)+1, \max \left(I_{n}\right)+2\right.$, $\left.\ldots, \max \left(I_{n}\right)+2^{3^{n+2}}\right\}$.

Second, the enumeration of the set $\bar{E}$ will be done with movable markers $m_{0}, m_{1}, \ldots$ such that $m_{e}$ sits at the beginning on the interval $I_{e}$ and it might be updated later, whenever necessary and permitted by $D$. On each interval can only sit one marker and it can only move from smaller to larger intervals. Each marker $m_{e}$ is associated with an $e$-state which is the value $d$ of the sum

$$
d=3^{e} \cdot \sum_{n=0,1, \ldots, e} a_{n} \cdot 3^{-n}
$$

where $a_{1}, a_{2}, \ldots, a_{e} \in\{0,1,2\}$. It is said that at the beginning of stage $s$ the marker $m_{e}$ is in the $e$-state $d$ iff

- there are exactly $2^{3^{e+1}-d}$ elements in $I_{k} \cap E_{s}$ where $I_{k}$ is the current interval on which $m_{e}$ sits;

- if $n \in\{0,1, \ldots, e\}$ and $a_{n} \in\{1,2\}$ then $\varphi_{n}(x)$ is defined on all values $x \leq \max \left(I_{e}\right)$ and $\varphi_{n}(x)+1=a_{n}$ for the $x \in I_{n} \cap E_{s}$.

The co-enumeration process of $E$ is done such that the following constraints are guaranteed.

- At the beginning of each stage $s$ and for each $k<s$, some marker $m_{e}$ sits on the interval $I_{k}$ iff $I_{k} \cap E_{s} \neq \emptyset$.

- All intervals $I_{k}$ with $k \geq s$ are subsets of $E_{s}$.

- If $m_{e}$ sits on $I_{k}$ with $k<s$ then $m_{e}$ is in some $e$-state $d$.

- At stage $s$, one searches for the lowest interval $I_{k}$ such that there is some $d \in D_{s+1}-D_{s}$ with $d \leq k$ permitting the update and the marker $m_{e}$ can increase its $e$-state by moving to some interval $I_{\ell}$ with $k \leq \ell \leq s$ and enumerating suitably many elements of $I_{\ell}$ into $\bar{E}$.

- If such $I_{k}, m_{e}$ are found, so that $m_{e}$ moves such that its $e$-state takes the highest possible $e$-state on some corresponding $I_{\ell}$ which it can obtain there by stage $s+1$. If necessary, some elements of $I_{\ell}$ are enumerated into $\bar{E}$. Furthermore, all intervals $I_{h}$ with $h \in$ $\{k, k+1, \ldots, s\}-\{\ell\}$ have all their elements enumerated into $\bar{E}$ by step $s+1$. For $t=1,2, \ldots$, the marker $m_{e+t}$ is moved to the interval $I_{s+t}$ in step $s$. 
- If such $I_{k}, m_{e}$ are not found, then no change is done in step $s$ and the construction directly goes on to stage $s+1$.

The main difference to the construction of a maximal set below $D$ with moveable markers are the following:

- Intervals $I_{k}$ are used in place of numbers $k$ and several elements are kept inside $E$ instead of one in the case of constructing cohesive sets; nevertheless, whenever the $e$-state goes up by changing an $a_{n}$ from 0 to 1 or 2 , the number of elements to be kept halves so that one can go for one of the sets $\left\{x: \varphi_{n}(x)+1=1\right\}$ and $\left\{x: \varphi_{n}(x)+1=2\right\}$.

- The idea to move all higher markers $m_{e+1}, m_{e+2}, \ldots$ is used to make $E$ introreducible. This idea comes from the construction of a frequency computable maximal set [17, Theorem 4.4]. But these authors have not yet seen this idea in the context of constructing $r$ maximal co-introreducible sets below a given high enumerable degree.

It is immediate to see that $\bar{E}$ is enumerable. Furthermore, $E \leq_{T} D$ by permitting. Also, every marker comes eventually to a rest and $E$ is infinite. As every interval $I_{k}$ contains either no or at least 2 elements of $E$, the enumerable set

$$
G=\left\{x: \exists k, s\left(x=\min \left(E_{s} \cap I_{y}\right)\right)\right\}
$$

and for every $k$ which intersects $E, I_{k} \cap E \cap G$ and $I_{k} \cap E \cap \bar{G}$ contain both at least one element. Hence $G$ and $\bar{G}$ have both an infinite meet with $E$ and $E$ is not cohesive.

It can be seen as follows that the set $E$ is introreducible. Let $F$ be an infinite subset of $E$ and let $I_{k}$ be any interval. Given $k$, one can find numbers $i, j$ such that $k<i<j$ and $F$ intersects both intervals $I_{i}$ and $I_{j}$. By construction, $E$ does not change on $I_{k}$ after stage $j$ as otherwise either $I_{i}$ or $I_{j}$ would be disjoint to $E$ and therefore also disjoint to $F$. So $E(x)=E_{j+1}(x)$ for all $x \in I_{k}$. Hence $E \leq_{T} F$.

The verification that $E$ is r-cohesive is similar to the usual methods to verify that a set constructed with markers going on numbers instead of intervals is co-maximal. For every rational number $q$ such that infinitely many markers $m_{e}$ end up in an $e$-state larger than $q \cdot 3^{e+1}$, one can define for every number $k$ the first number $s$ such that either $I_{k} \subseteq E_{s}$ or there is an $\ell$ with $k \leq \ell \leq s$ and a marker $m_{e}$ on $I_{k}$ which could in stage $s$ move onto $I_{\ell}$ in a way that its $e$-state is afterwards larger than $q \cdot 3^{e+1}$. Then this function is defined on all up to finitely many numbers $k$ and for all but finitely many $k$ there is a stage $t>s$ for the considered $s$ such that $D$ would permit either permit some marker $m_{e^{\prime}}$ sitting on $I_{k}$ at $t$ to go into an $e$-stage larger than $q \cdot 3^{e+1}$ or $I_{k} \cap E_{t}=\emptyset$.

Theorem 20. Given natural numbers $m, n$ such that $m$ is even and $1 \leq$ $n \leq m$, the following three statements are equivalent for every $n$-enumerable set $A$. 
(1) There is an enumerable set $E \leq_{T} A$ such that $E$ has high Turing degree;

(2) There is an m-enumerable and dense immune set in the Turing degree of $A$;

(3) There is an m-enumerable and $r$-cohesive set in the Turing degree of $A$.

Proof. $(1 \Rightarrow 2)$ : As there is a high enumerable set below $A$, there is also a dense immune set $E \leq_{T} A$ such that $E$ is co-enumerable and retraceable. Then $E$ is also introreducible as every dense immune set is introreducible.

As $E$ is retraceable, $E$ is not hyperhyperimmune. Thus there is a weak disjoint array $V_{0}, V_{1}, V_{2}, \ldots$ intersecting $\bar{E}$. One can create a further array $U_{0}, U_{1}, U_{2}, \ldots$ such that

$$
U_{x} \subseteq V_{x^{2}} \cup V_{x^{2}+1} \cup \ldots \cup V_{x^{2}+2 x}-\{0,1,2, \ldots, x-1\}
$$

and $\left|U_{x}-E\right|=1$ for all $x$. The unique element $f(x) \in U_{x}-E$ can be computed by some function $f \leq_{T} E$. The set

$$
B=\{f(x): x \in A\}
$$

is $m$-enumerable as $m \geq n$ and $m$ is even. To see this, let $A_{s}$ be an $n$ enumeration of $A$ (which is also an $m$-enumeration as $m \geq n$ ), $E_{s}$ be a co-enumeration of $E$ and $U_{x, s}$ be a uniform enumeration of the $U_{x}$.

Now a number $u$ belongs to $B_{s}$ iff $u \in E_{s}$ and there is an $x \leq s$ with $u \in U_{x, s} \wedge x \in A_{s}$. This $x$, if it exists, is unique. One can see that the first time $u$ enters $B$ needs that $A$ makes a mind change at $x$ up to stage $s$ as well. Furthermore, the $m$-th mind change of $A$ at $x$ is that $x$ goes out of $A$ as $m$ is even; thus forcing $u$ out of $B$ by enumerating $u$ into the complement of $E$ can only cause an additional mind change if $A$ has not yet done all its $m$ mind changes on $x$; thus $B$ is $m$-enumerable.

As $f \leq_{T} E$ and $E \leq_{T} A$, one can check relative to $A$ for each $x, y$ whether $y=f(x)$. Furthermore, $f(x) \geq x$ by the choice of the $U_{0}, U_{1}, \ldots$, thus $y \in B$ iff there is $x \leq y$ with $x \in A$ and $y=f(x)$. Hence $B \leq_{T} A$.

As $E$ is introreducible and $B$ an infinite subset of $E, E \leq_{T} B$ and $f \leq_{T} B$. As $A(x)=B(f(x))$ for all $x, A \leq_{T} B$.

$(1 \Rightarrow 3)$ : Let $E \leq_{T} A$ be a set which is introreducible, r-cohesive, not cohesive and co-enumerable. This set exists by Proposition 19. But then $E$ is not hyperhyperimmune, as every r-maximal hyperhypersimple set is maximal [28, Proposition X.4.5]. Thus there is a weak disjoint array intersecting $E$ and one can construct a further weak array $U_{0}, U_{1}, U_{2}, \ldots$ in the same way as in the part $(1 \Rightarrow 2)$. Again there is $f \leq_{T} E$ such that $\{f(x)\}=U_{x} \cap E$ and $f(x) \geq x$ for all $x$. Now the set

$$
C=\{f(x): x \in A\}
$$

is r-cohesive as it is a subset of an r-cohesive set. One can argue as in the part $(1 \Rightarrow 2)$ that $C \equiv_{T} A$ and $C$ is $m$-enumerable. 
$(2 \Rightarrow 1)$ : Assume now that $A$ is Turing equivalent to a dense immune set $B$ and let $k$ be the least number such that $B$ is $k$-enumerable. Now let $f$ be a one-to-one function such that the range of $f$ contains exactly those $x$ on which the $k$-approximation of $B$ makes at least $k-1$ mind changes. Note that the intersection of $B$ and the range of $f$ is infinite; indeed $f(y) \in B$ iff $B$ changes on $f(y)$ its mind $k-1$ but not $k$ times. Let

$$
E=\{y: f(y) \notin B\} .
$$

This set is enumerable. Consider any computable function $g$ and any $x$. If $\{e \notin E: e \leq g(x)\}$ has at least $x$ elements, then $\{b \in B: b \leq \max \{f(y)$ : $y \leq g(x)\}\}$ has also at least $x$ elements. But as $B$ is dense immune, this happens only for finitely many $x$. Hence $E$ is dense simple. As the Turing degree of a dense simple set is high, $A$ bounds a high enumerable set.

$(3 \Rightarrow 1)$ : The first part is parallel to $(2 \Rightarrow 1)$. Assume now that $A$ is Turing equivalent to a $r$-cohesive set $C$ in the Ershov hierarchy and let $k$ be the least number such that $C$ is $k$-enumerable. Now let $f$ be a one-toone function such that the range of $f$ contains exactly those $x$ on which the $k$-approximation of $C$ makes at least $k-1$ mind changes. Note that the intersection of $C$ and the range of $f$ is infinite; indeed $f(y) \in C$ iff $C$ changes on $f(y)$ its mind $k-1$ but not $k$ times. Now consider the set $E=\{y: f(y) \notin C\}$. This set is enumerable. If the set $E$ has high Turing degree, then the proof would be completed.

So, for the remaining part of the proof, assume by way of contradiction that $E$ does not have high Turing degree. Let $E_{0}, E_{1}, E_{2}, \ldots$ be an effective enumeration of $E$ and let $e_{0}, e_{1}, e_{2}, \ldots$ be the complement of $E$ in ascending order. Since $E$ is not high, there is a computable and increasing function $g$ such that, for infinitely many $x$,

$$
g(x)>e_{10 x} \text { and } E(y)=E_{g(x)}(y) \text { for all } y \leq e_{10 x} .
$$

Let $x \sim x^{\prime}$ denote that there are $y, y^{\prime} \leq z$ where $z=\max \left\{g(x), g\left(x^{\prime}\right)\right\}$, $f(y)=x, f\left(y^{\prime}\right)=x^{\prime}, y \notin E_{z}, y^{\prime} \notin E_{z}$ but $u \in E_{z}$ for all $u$ with $\min \left\{y, y^{\prime}\right\}<$ $u<\max \left\{y, y^{\prime}\right\}$. Now define the following set $D$ by

$$
D(v)= \begin{cases}1-D(w) & \text { for the largest } w<v \text { with } v \sim w \\ 0 & \text { if there is no such } w .\end{cases}
$$

The set $D$ is decidable as the functions $f, g$ and the relation $\sim$ are computable.

Given any lower bound $b$, there is an $x>b$ such that $x$ satisfies Condition (4). There is then an $y<10 x-3$ such that

$$
x<\min \left\{f\left(e_{y}\right), f\left(e_{y+1}\right), f\left(e_{y+2}\right), f\left(e_{y+3}\right)\right\} .
$$

Note that $f\left(e_{y}\right) \sim f\left(e_{y+1}\right) \sim f\left(e_{y+2}\right) \sim f\left(e_{y+3}\right)$ by Condition (4). Let $v=$ $\max \left\{f\left(e_{y+1}\right), f\left(e_{y+2}\right)\right\}$. Then the $w$ in the case distinction for the definition of $v$ exists and is an element of $\left\{f\left(e_{y}\right), f\left(e_{y+1}\right), f\left(e_{y+2}\right), f\left(e_{y+3}\right)\right\}-\{v\}$. It follows that $D(v)$ is different from $D(w)$. As $f\left(e_{0}\right), f\left(e_{1}\right), f\left(e_{2}\right), \ldots \in C$, 
one gets the desired contradiction: Above every bound $b$ there are $v, w \in C$ with $D(v) \neq D(w)$. This contradicts to the assumption that $C$ is $r$-cohesive. As a consequence, $E$ must in the above case be high and $A$ bounds a high enumerable set.

A parallel result can be obtained in an attempt to characterise the low $_{2}$ degrees. Here again, the characterisation does not give those $n$-enumerable degrees which are low 2 but those which bound only low 2 enumerable degrees.

Theorem 21. Let $A$ be a set in the Ershov hierarchy. Then the following are equivalent:

(1) Every enumerable set $B \leq_{T} A$ is low ;

(2) For every $n$ and every $B \equiv_{T} A$ which is n-enumerable there are maximal sets $M, N$ with $\bar{M} \subseteq B \subseteq N$.

Proof. Assume that there is an enumerable set $B \leq_{T} A$ which is not low 2 . Soare [28, Theorem XI.4.1] gives the proof of a theorem of Shoenfield by showing that Dekker's deficiency set $E$ of $B$ has no maximal superset. This set $E$ has a retraceable complement $\left\{e_{0}, e_{1}, e_{2}, \ldots\right\}$; in particular, the mapping $e_{n} \mapsto n$ has a total computable extension. Now the set

$$
E_{A}=\left\{e_{n}: n \in A\right\} \cup E
$$

is in Ershov's difference hierarchy, Turing equivalent to $A$ and has no maximal superset.

For the other direction, assume that all enumerable sets below $A$ are $\operatorname{low}_{2}$. Now let $B \equiv_{T} A$ be given and let $n$ be the least number such that $B$ is $n$ enumerable; $n>1$ since otherwise there is nothing to prove by Lachlan's result [28, Theorem XI.5.1]. There is a one-to-one computable function $f$ such that its range contains all $x$ on which some fixed $n$-approximation to $B$ changes its mind at least $n-1$ times. The set

$$
E=\{y: \text { the approximation to } B \text { changes on } f(y) \text { its mind } n \text { times }\}
$$

is enumerable, Turing reducible to $A$ and thus low 2 . Thus $E$ has a maximal superset $U$. The set $\{f(u): u \notin U\}$ is cohesive and 2-enumerable: a suitable approximation is 0 on any $x$ until a $y$ with $f(y)=x$ is found. Then the approximation goes to 1 and returns to 0 iff $y$ is enumerated into $U$. By Theorem 5 , the set $U$ is the complement of a maximal set $N$. Furthermore, the set of all $x$ where the $n$-approximation of $B$ makes $n$ mind changes is enumerable and thus has a co-maximal subset, let $M$ be the complement of this subset. Then $\bar{M} \subseteq B \subseteq N$ in the case that $n$ is odd and $\bar{N} \subseteq B \subseteq M$ in the case that $n$ is even.

Ishmukhametov and $\mathrm{Wu}[16]$ have shown that there is a high properly 2enumerable degree such that all enumerable degrees bounded by it are low. Indeed, this degree is even isolated in the terminology of Cooper and $\mathrm{Yi}$ [8] as there is a largest enumerable degree below it which is low. Then, by Theorems 20 and 21, this degree, although high, behaves as it would low 2 . 
Thus, this degree witnesses that condition (1) in Theorems 20 and 21 has indeed to refer to the enumerable degrees below $A$ and not to the degree of $A$ itself. This is formalized in the following corollary.

Corollary 22. There exists a high 2-enumerable degree which contains no dense immune or r-cohesive n-enumerable set for any $n$. Furthermore, for every $n$ and every $n$-enumerable set $B$ in this degree there are maximal sets $M, N$ with $\bar{M} \subseteq B \subseteq N$.

Theorem 5 states that every cohesive set in the Ershov hierarchy is actually co-enumerable. The next result shows that, in contrast to this, r-cohesive sets in the Ershov hierarchy can have proper 2-enumerable degree.

Corollary 23. There exist high properly 2-enumerable degrees which contain both, an 2-enumerable dense-immune and a 2-enumerable r-cohesive set.

Proof. For instance, one can use the Cooper-Lempp-Watson Density Theorem [7] to get properly 2-enumerable degrees which bound high enumerable degrees. Then the result follows from Martin's Theorem [20] and Theorem 20 above.

\section{REFERENCES}

[1] Bahareh Afshari, George Barmpalias and S. Barry Cooper, Immunity Properties and the $n$-C.E. Hierarchy, in Theory and Applications of Models of Computation, Third International Conference on Computation and Logic, TAMC 2006, Beijing, May 2006, Proceedings, (Jin-Yi Cai, S. Barry Cooper, Angsheng Li, eds.), Springer Lecture Notes in Computer Science, 3959, 694-703, 2006.

[2] George Barmpalias, Computability and applications to Analysis, PhD thesis, University of Leeds, U.K., 2004.

[3] George Barmpalias, Hypersimplicity and Semicomputability in the Weak Truth Table Degrees, Archive for Math. Logic 44, 1045-1065, 2005.

[4] George Barmpalias and Andrew Lewis, The Hypersimple-free c.e. wtt degrees are dense in the c.e. wtt degrees, Notre Dame Journal of Formal Logic Volume 47 Issue 3 (2006).

[5] Mark Bickford and Charlie F. Mills, Lowness properties of r.e. sets (unpublished manuscript), UW Madison, 1982.

[6] S. Barry Cooper, Jump equivalence of the $\Delta_{2}^{0}$ hyperhyperimmune sets, The Journal of Symbolic Logic, 37, 598-600, 1972.

[7] S. Barry Cooper, Steffen Lempp and Philip Watson, Weak density and cupping in the d-r.e. degrees, Israel Journal of Mathematics, 67, 137-152, 1989.

[8] S. Barry Cooper and Xiaoding Yi, Isolated d.r.e. degrees, unpublished.

[9] S. Barry Cooper, Computability Theory, Chapman \& Hall/ CRC Press, Boca Raton, FL, New York, London, 2004.

[10] Nigel J. Cutland, Computability, an introduction to recursive function theory, Cambridge University Press, 1980.

[11] Rodney Downey and Denis Hirschfeldt, Algorithmic Randomness and Complexity, Manuscript on Internetpage 〈http://www.mcs.vuw.ac.nz/ ${ }^{\sim}$ downey/ $\rangle$.

[12] Rodney G. Downey and Carl G. Jockusch, Jr., T-degrees, jump classes and strong reducibilities, Transactions of the American Mathematical Society, 301, 103-136, 1987.

[13] Rodney G. Downey and Steffen Lempp, Contiguity and distribuity in the enumerable Turing degrees, The Journal of Symbolic Logic, 62, 1215-1240, 1997. 
[14] Santiago Figueira, André Nies and Frank Stephan, Lowness properties and approximations of the jump, Annals of Pure Applied Logic, to appear; also available as Technical Report TR11/05, School of Computing, National University of Singapore, 2005.

[15] Shamil Ishmukhametov, Weak recursive degrees and a problem of Spector, Recursion theory and complexity. Proceedings of the Kazan 1997 Workshop, Kazan, Russia, July 14-19, 1997. Walter de Gruyter, Berlin, pages 81-87, 1999.

[16] Shamil Ishmukhametov and Guohua Wu, Isolation and the high/low hierarchy, Archive of Mathematical Logic, 41, 259-266, 2002.

[17] Martin Kummer and Frank Stephan, Recursion theoretic properties of frequency computation and bounded queries, Information and Computation, 120, 59-77, 1995.

[18] Alistair H. Lachlan, On the lattice of recursively enumerable sets, Transactions of the American Mathematical Society, 130, 1-37, 1968.

[19] Donald A. Martin, A theorem on hyperhypersimple sets. The Journal of Symbolic Logic, 28, 273-278, 1963.

[20] Donald A. Martin, Classes of recursively enumerable sets and degrees of unsolvability, Zeitschrift für Mathematische Logik und Grundlagen der Mathematik, 12, 295-310, 1966.

[21] André Nies, Reals that compute little, Proceedings of Logic Colloquium 2002, Lecture Notes in Logic, 27, 261-275, 2006.

[22] Piergiorgio Odifreddi, Classical recursion theory, Volumes I and II, North-Holland, Amsterdam, Oxford, 1989 and 1999.

[23] James C. Owings, Recursion, metarecursion and inclusion, The Journal of Symbolic Logic, 32, 173-178, 1967.

[24] Emil L. Post, Recursively enumerable sets of positive integers and their decision problems, Bulletin of the American Mathematical Society, 50, 284-316, 1944.

[25] Julia Robinson, Recursive functions of one variable, Proceedings of the American Mathematical Society, 19, 815-820, 1968.

[26] Leonard P. Sasso, Jr., Deficiency sets and bounded information reducibilities, Transactions of the American Mathematical Society 200, 267-290, 1974.

[27] Joseph R. Shoenfield, Degrees of classes of r.e. sets, The Journal of Symbolic Logic, 41, 695-696, 1976.

[28] Robert I. Soare, Recursively enumerable sets and degrees, Springer-Verlag, Berlin, London, 1987.

[29] Liang Yu and Yue Yang, On the definable ideal generated by nonbounding c.e. degrees, The Journal of Symbolic Logic 70 Number 1, (2005)

\section{AdDRESSES}

Bahareh Afshari. School of Mathematics, University of Leeds, Leeds, LS2 9JT, U.K.; Email: bahareh@maths.leeds.ac.uk.

George Barmpalias. School of Mathematics, University of Leeds, Leeds, LS2 9JT, U.K.; Email: georgeb@maths.leeds.ac.uk.

S. Barry Cooper. School of Mathematics, University of Leeds, Leeds, LS2 9JT, U.K.; Email: pmt6sbc@leeds.ac.uk.

Frank Stephan. Departments of Mathematics and Computer Science, National University of Singapore, 2 Science Drive 2, Singapore 117543, Republic of Singapore; Email: fstephan@comp.nus.edu.sg. 